\title{
NEONATAL HYPERTONIA AND MATERNAL SMOKING
}

A detailed neurologic examination was performed in 6-week-old infants enrolled in a study of effects of in utero cocaine exposure, while controlling for concomitant cigarette smoking exposure and other variables, and results are reported from the University of California, San Francisco, CA. Examination of 40 cocaine-exposed compared to 56 unexposed infants showed that hypertonia correlated with maternal cigarette smoking during pregnancy but not with in utero cocaine exposure. Using maternal urine conitine levels as a measure of nicotine exposure, the odds ratio for an abnormal neurologic examination was 2.9, if maternal urine conitine was $>200 \mathrm{ng} / \mathrm{ml}$. As the concentration of cotinine increased, the risk of hypertonia increased. Maternal cigarette smoking may be the major predictor of abnormal hypertonia reported in cocaine-exposed infants. (Dempsey DA, Hajnal BL, Partridge JC et al. Tone abnormalities are associated with maternal cigarette smoking during pregnancy in in utero cocaine-exposed infants. Pediatrics July 2000;106:79-85). (Reprints: Delia A Dempsey MD, Clinical Pharmacology, Box 0898 CRM, University of California, San Francisco, CA 94143).

COMMENT. Neonatal hypertonia, commonly reported among in utero cocaine-exposed infants, is correlated with maternal cigarette smoking during pregnancy, and is not caused by the cocaine exposure per se. This generalized hypertonia is found to resolve by 2 years of age, and is not predictive of later development of cerebral palsy. It may be a marker for adverse childhood cognitive or behavioral disorders such as ADHD, complications previously reported in association with prenatal exposure to tobacco smoke or cocaine.

Effects of cocaine and cocaine + alcohol on cognitive function of adults. Cocaine and alcohol each selectively and negatively affect neurocognitive performance of neurobehavioral tests after 1 to 3 days forced abstinence. The effects are found to persist after 4 weeks of abstinence. The concomitant abuse of cocaine and alcohol has additive negative effects on brain function. (Bolla KI, Funderburk FR, Cadet JL. Neurology 2000;54:2285-2292).

\section{CHARCOT-MARIE-TOOTH DISEASE TYPE 1A: AXONAL LESIONS}

The clinical and electrophysiological phenotype in 42 patients with Charcot-Marie-Tooth disease type 1A (CMT1A) were analysed at Wayne State University, Detroit, MI. Muscle weakness, and reduced amplitudes and motor unit number estimates of compound motor nerve action potentials (CMAPs) correlated with clinical disability, while slowed nerve conduction velocities (NCVs) fid not. Loss of joint position sense and reduction in amplitude of sensory nerve action potentials (SNAPs) also correlated with disability, while sensory NCVs did not. Neurological dysfunction and disability in CMT1A are correlated with loss or degeneration of large motor and sensory axons. (Krajewski KM, Lewis RA, Fuerst DR et al. Neurological dysfunction and axonal degeneration in Charcot-MarieTooth disease type 1A. Brain July 2000;123:1516-1527). (Respond: Michael E Shy MD, Department of Neurology, Wayne State University School of Medicine, Elliman Building, Room 3301, 421 E Canfield, Detroit, MI 48201).

COMMENT. The clinical neurologic examination, EMG and NCS may be used to measure the degenerative process affecting axons in peripheral nerves in CMT1A, and any benefits from novel therapies to promote axonal regeneration in the future. 